\title{
Secondary Ion Mass Spectrometry on the Helium Ion Microscope: methodologies for analysis of nanomaterials
}

Jean-Nicolas Audinot ${ }^{1}$, Florian Vollnhals ${ }^{1}$, Paul Gratia ${ }^{1}$, Santhana Eswara ${ }^{1}$, Patrick Philipp ${ }^{1}$ and Tom Wirtz ${ }^{1}$

1. Advanced Instrumentation for Ion Nano-Analytics (AINA), MRT Department, Luxembourg Institute of Science and Technology (LIST), 41 rue du Brill, L-4422 Belvaux, Luxembourg

These last 2 decades, engineered nanomaterials have become the focus of intensive research in diverse areas, including cosmetics, textiles, electronics devices, materials engineering, energy production, surface cleaning, aerospace, and medical applications. The current trend in nanotechnology is the chemical and/or structural modification of materials so that they present specific desired properties such as reduction/increase of redox potential, modification of the conductive or/and ferroelectric properties, surface properties, etc. For this purpose, to allow the characterization of the nanomaterials, we added nano-analytical capabilities to the Helium Ion Microscope (HIM) by developing a Secondary Ion Mass Spectrometry (SIMS) system specifically designed for the Zeiss ORION NanoFab HIM [1-3]. SIMS is based on the generation and identification of characteristic secondary ions by irradiation with a primary ion beam. It is an extremely powerful technique for analysing surfaces owing in particular to its excellent sensitivity (detection limits down to the ppb are possible, so that SIMS can be used to detect both major and trace elements), high dynamic range (a same signal can be followed over several orders of magnitude), and ability to differentiate between isotopes.

In SIMS, the typical interaction volume between the impinging ion beam and the sample is around 10 $\mathrm{nm}$ in the lateral direction. As the probe size in the HIM is substantially smaller (both for He and $\mathrm{Ne}$ ), the SIMS lateral resolution on the integrated HIM-SIMS is limited only by fundamental considerations and not, as is currently the case on commercial SIMS instruments, the probe size [2-4]. We have demonstrated that our instrument is capable of producing elemental SIMS maps with lateral resolutions down to $12 \mathrm{~nm}$ [2-6]. Furthermore, HIM-SIMS opens the way for in-situ correlative imaging combining high resolution SE images with elemental and isotopic ratio maps from SIMS [2,3,7]. This approach allows SE images of exactly the same zone analyzed with SIMS to be acquired easily and rapidly, followed by a fusion between the SE and SIMS data sets. Moreover, with the SIMS add-on, it is now possible to follow the chemical composition in real time during nano-patterning in the HIM for applications such as end-pointing.

Here, we will review the instrument performance and present a number of examples taken from various fields of materials science (battery materials, solar cells, micro-electronics, coatings, multilayers) and life science (nanoparticles in creams and biological tissues) to show the powerful analytical capabilities and correlative microscopy possibilities enabled by the integrated HIM-SIMS instrument (figure 1). 


\section{References :}

[1] T. Wirtz, N. Vanhove, L. Pillatsch, D. Dowsett, S. Sijbrandij, J. Notte, Appl. Phys. Lett. 101 (4) (2012) 041601-1-041601-5

[2] T. Wirtz, D. Dowsett, P. Philipp, Helium Ion Microscopy, edited by G. Hlawacek, A. Gölzhäuser, Springer, 2017

[3] D. Dowsett, T. Wirtz, Anal. Chem. 89 (2017) 8957-8965

[4] T. Wirtz, P. Philipp, J.-N. Audinot, D. Dowsett, S. Eswara, Nanotechnology 26 (2015) 434001

[5] P. Gratia, G. Grancini, J.-N. Audinot, X. Jeanbourquin, E. Mosconi, I. Zimmermann, D. Dowsett, Y. Lee, M. Grätzel, F. De Angelis, K.Sivula, T. Wirtz, M. K. Nazeeruddin, J. Am. Chem. Soc. 138 (49) (2016) 15821-15824

[6] P. Gratia, I. Zimmermann, P. Schouwink, J.-H. Yum, J.-N. Audinot, K. Sivula, T. Wirtz, M. K. Nazeeruddin, ACS Energy Lett. 2 (2017) 2686-2693

[7] F. Vollnhals, J.-N. Audinot, T. Wirtz, M. Mercier-Bonin, I. Fourquaux, B. Schroeppel, U. Kraushaar, V. Lev-Ram, M. H. Ellisman, S. Eswara, Anal. Chem. 89 (2017) 10702-10710
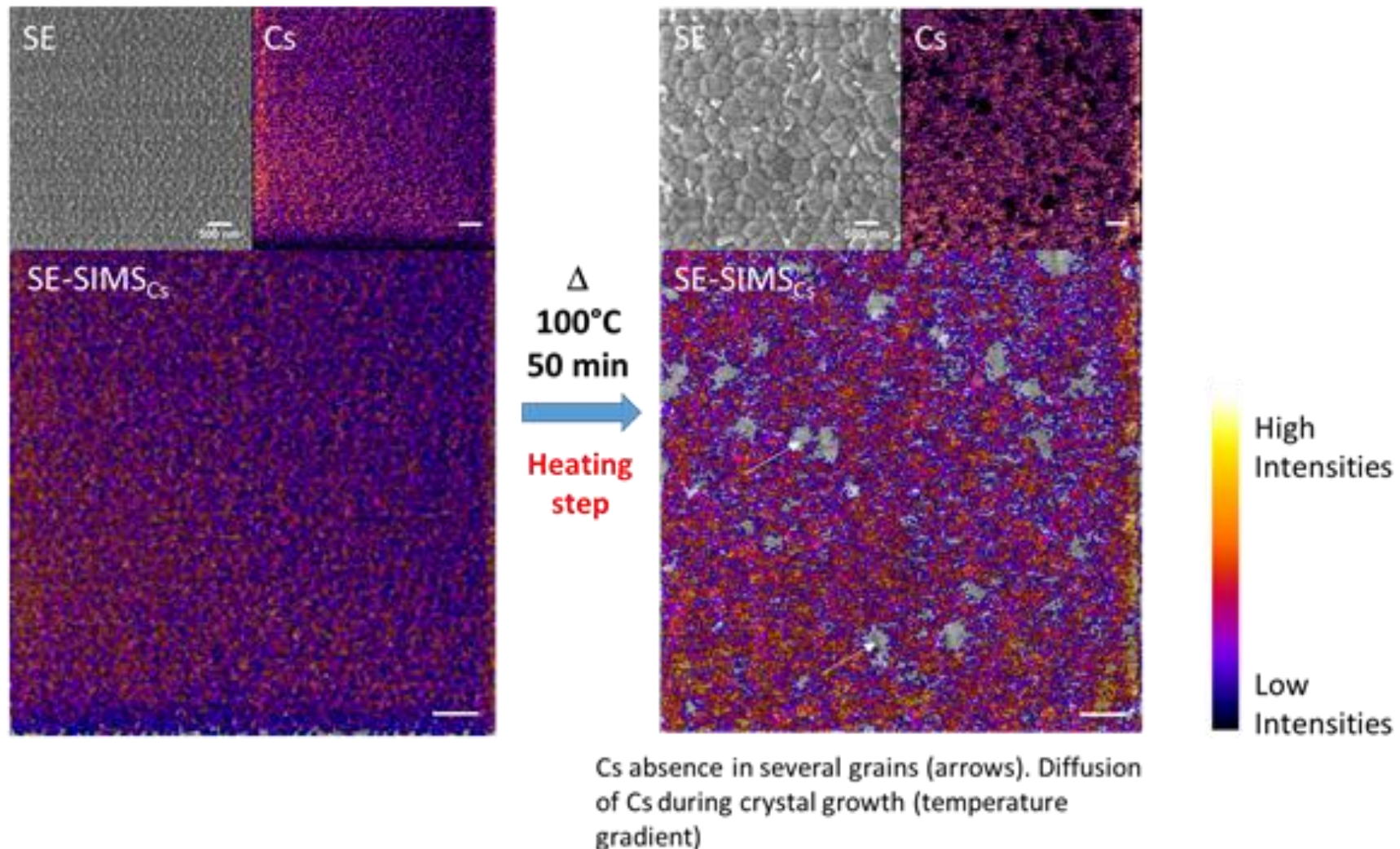

Figure 1: Comparison of a $\mathrm{Cs}(\mathrm{I} / \mathrm{Br})$ perovskite film before (vacuum-dried at room temperature) and after annealing for $50 \mathrm{~min}$ at $100{ }^{\circ} \mathrm{C}$. Secondary electron (SE) images from helium ion microscopy (HIM). Secondary ion mass spectrometry (SIMS) imaging of Cs. Combined SIMS and HIM imaging according to the methodology presented in [7] correlating local variations in Cs with grain morphology. The scale bar corresponds to $500 \mathrm{~nm}$ in all of the images. Cs absence in several grains is clearly observed [6]. 